\title{
On the Capacity of Packet Reservation Multiple Access with Capture in Personal Communication Systems
}

\author{
Xiaoxin Qiu, Member, IEEE, and Victor O. K. Li, Fellow, IEEE
}

\begin{abstract}
In this paper, a new Markovian model is developed to analyze the Packet Reservation Multiple Access (PRMA) protocol over Rician fading channels with lognormal shadowing. The packet dropping probability and the voice user capacity are defined as the performance measures. A new formula is derived to evaluate the dropping probability in the fading environment. The analytical results from our new formula show close agreement with those from computer simulations. The capture effect on the PRMA system performance is also studied. We found that, with capture, the user capacity will be improved and the service quality will degrade gracefully when the number of active users in the system increases. Using the dropping probability as the criterion, we also determine the optimal permission probability in a microcellular environment.
\end{abstract}

\section{INTRODUCTION}

$\mathbf{P}$ ACKET reservation multiple access (PRMA) has received much attention in personal communications systems (PCS) because of its efficiency and flexibility. It combines random access with time division access, which greatly improves the spectrum efficiency and the system capacity compared to just using random access or time division access alone.

The performance of PRMA in the noiseless environment has been studied intensively [1], [2], [7]. Since wireless systems suffer multipath fading and shadowing, however, the results derived in the above papers will be too optimistic. In [3], the performance of PRMA has been simulated with fixed packet header error rate. It is found [3] that when the packet header error rate is large, i.e., the channel is very noisy, the system performance will be much worse than that with perfect channels. To study the performance of PRMA in fading channels analytically, a mathematical model is developed in [9]. The results given by this model, however, do not fit the simulations very well, especially when the packet header error rate is large.

In this paper, we propose a modified Markovian model to analyze the performance of PRMA in a microcellular environment. We use the dropping probability and the voice user capacity as the performance measures. A new formula is derived to evaluate the dropping probability in the presence of

Manuscript received March 1, 1994; revised October 27, 1994 and February 23, 1996 .

The authors are with the Communication Sciences Institute, Department of Electrical Engineering, University of Southern California, Los Angeles, CA 90089-2565 USA.

Publisher Item Identifier S 0018-9545(96)08198-4. transmission errors. The voice user capacity is redetermined under new conditions. We also study the impact of capture on the PRMA performance. We modify the methodology developed for Rayleigh fading in [5] and [10] to calculate the bit error rate and the capture probability in the shadowed Rician fading channel. Our objective is to study the PRMA system behavior in the PCS environment, accounting for both the physical layer and the data link layer. Our results indicate that, with capture, PRMA exhibits the desirable soft capacity feature.

The rest of the paper is organized as follows. In Section II, the PRMA protocol is briefly described. The system performance evaluation model is developed and a new dropping probability formula is derived in Section III. The capture model is developed in Section IV. In Section V, numerical results are given. Finally, we conclude in Section VI.

\section{PRMA PROTOCOL}

We consider a voice-only system in this paper. It is well known that a voice conversation is made up of silence gaps and talkspurts. During the silence gap, no voice packet will be generated. The resource can be released to other users to obtain higher multiplexing efficiency. The voice activity detector is used in PRMA to detect silence gaps.

In PRMA, time is divided into slots that are grouped into frames. These slots can be reserved by users. Once a slot is reserved, we call it unavailable. Otherwise, it is available. The availability of slots is tracked by the base station and this information is broadcast to users at the end of each slot. If a voice user has packets to transmit, he needs to select an available slot and try to make a reservation by transmitting at that slot. If the reservation succeeds, this slot will be used by him in subsequent frames until the end of the talkspurt. Then, the slot will be released and be available again. Since voice communication requires prompt delivery, the voice packet will be discarded if it cannot be transmitted within a delay limit. Once a packet is dropped, the subsequent ones in the talkspurt will continue to attempt to make a successful reservation [1], [7]. The capture mechanism is an option in the PRMA system. With capture, the system performance can be improved, but at the expense of more complexity.

At the end of each slot, the base station broadcasts a short feedback message in the down link. Two kinds of information will be fed back: idle and reserved, with the Identification 
Number of the reserving user (if there is any). In the fading environment, there are two reasons for an idle feedback, namely, no transmission and unsuccessful transmission. The unsuccessful transmission may be due to collision or channel noise or both. For a system without capture, all the transmissions will fail if a collision occurs. For a system with capture, however, the stronger packet may still survive even though there is a collision. Dropped packets due to collision is the only reason for the degraded performance in perfect channels. But in a fading environment, the channel noise may cause decoding errors at the receiver whether there is a collision or not. Therefore, the channel noise (fading, shadowing, interference, etc.) is another important reason for unsuccessful reservation and further degradation of the system performance.

In PRMA, each terminal is assigned a permission probability $P_{t}$. The user transmission is controlled by this parameter. To get a reserved feedback, i.e., make a successful reservation, the slot should be idle, the terminal should have permission to transmit, and the receiver should decode this packet correctly [3], [9]. In perfect channels, after the successful reservation, the user will keep this slot until the end of the talkspurt. But in the presence of transmission errors, the reserved slot may be lost before the talkspurt ends, thus causing higher dropping probability and worse service quality compared to a system with perfect channels [3].

\section{Performance Evaluation Model}

In this paper, we assume that the frequencies are reused in the system. The frequency reuse distance is long enough so that the cochannel interference can be ignored. This assumption is made only for computational simplicity. The following analysis can be easily extended to account for the effect of cochannel interference. We focus on one cell and assume each cell will behave statistically in the same way. It is also assumed that the system is stationary and the state transition occurs only at the end of each slot.

The dropping probability and the voice user capacity will be used as the performance measures. The dropping probability is defined as the portion of dropped packets in a talkspurt. The voice user capacity is the number of voice users which can be supported under certain dropping probability constraints.

\section{A. Terminal Model}

In PRMA, the active user may be in one of three states, namely, 1) the silence state $(S), 2)$ the reservation state (R), and 3 ) the contention state (C). The transition among these three states is illustrated in Fig. 1. In this figure, $\gamma_{f}$ is the probability that a talkspurt ends in a frame; $u$ is the probability that a talkspurt ends in a time slot; $P_{C R}$ is the probability that a terminal gets the reservation in the current time slot; $P_{R C}$ is the probability that a terminal loses the reservation in the current slot because of transmission errors; and $P_{S C}$ is the probability that the silence gap ends during a time slot.

It is commonly assumed that the lengths of the talkspurts and the silence gaps are exponentially distributed. Let $t_{1}$ and $t_{2}$ denote the average lengths of talkspurts and silence gaps,

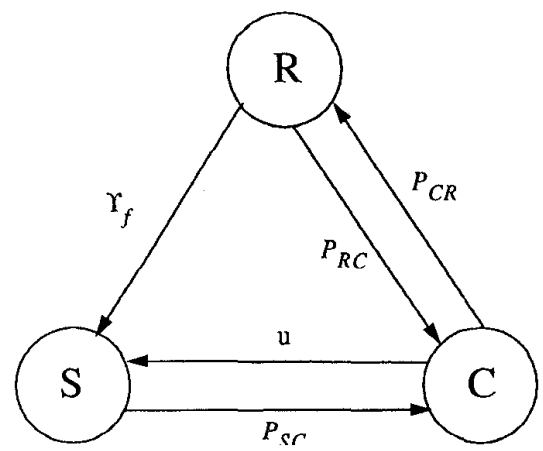

Fig. 1. PRMA terminal model.

respectively. Then

$$
\begin{aligned}
P_{S C} & =1-\exp \left(\frac{-\tau}{t_{2}}\right) \\
u & =1-\exp \left(\frac{-\tau}{t_{1}}\right) \\
\gamma_{f} & =1-(1-u)^{N} \\
P_{R C} & =\sigma\left(1-\gamma_{f}\right)
\end{aligned}
$$

where $\tau$ is the duration of a slot; $\sigma$ is the packet header error rate; and $N$ is the number of slots per frame. The value of $P_{C R}$ depends on the system characteristics such as capture probability. It will be evaluated in the following sections.

\section{B. System Model}

We consider a sufficiently large number of sources multiplexed on the same channel, so that the resultant composite process of the state-change events will be close to a Poisson one [6]. As in [9], the PRMA system can be described by three state variables, $\left\{N_{R}, N_{S}, N_{C}\right\}$, which are the number of terminals in the reservation state, in the silence state, and in the contention state, respectively. Denote the number of active terminals in a cell as $M$. Then

$$
N_{R}+N_{S}+N_{C}=M \quad \text { and } \quad 0 \leq N_{R} \leq N .
$$

With the above condition, only two of these three state variables are independent. Here, we choose $N_{R}$ and $N_{S}$ as independent state variables. To study a two-variable system, a two-dimensional (2-D) Markov chain is usually needed. By exploiting the special feature of PRMA, we can simplify the 2D Markov chain to two one-dimensional (1-D) Markov chains, thus greatly reducing the computational complexity [6], [9].

PRMA can be described by two evolving processes: the speaking-silence process and the reservation process [6]. We observe that the state transition of the speaking-silence process evolves independently of the individual values of $N_{R}$ and $N_{C}$. It can be described completely by the value of $N_{S}$. With this observation, the 2-D Markovian model can be simplified to two 1-D Markovian models, namely, the model of the speaking silence process and the model of the reservation process. State transition diagrams of these two processes are shown in Figs. 2 and 3 , respectively.

In Fig. 2, the speaking-silence process is illustrated. The state variable is the number of users in silence, i.e., $N_{S}$. In 


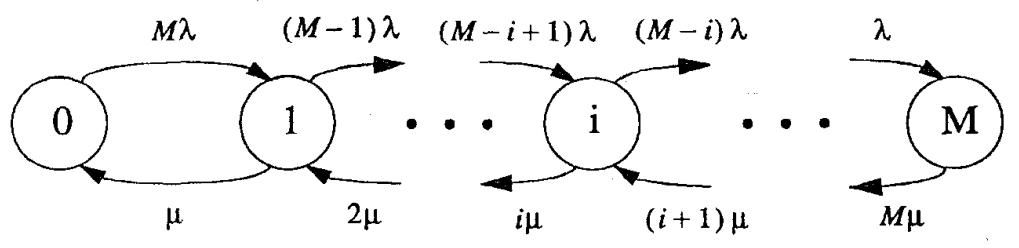

Fig. 2. State transition diagram of speaking-silence process.

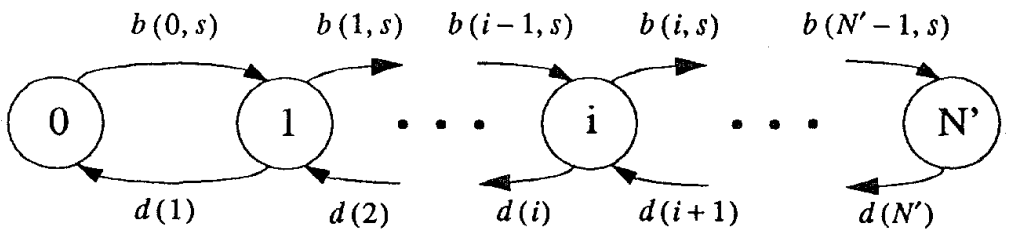

Fig. 3. State transition diagram of reservation process.

this figure, $\lambda=1 / t_{1}$ and $\mu=1 / t_{2}$. Define $\rho=\lambda / \mu$. It is an $M / M / m / / m$ queue, with $m$ being the number of active users. Solving this Markov chain, we get [4]

$$
P\left(N_{S}=i\right)=\left(\begin{array}{c}
M \\
i
\end{array}\right) \frac{\rho^{i}}{(1+\rho)^{M}}
$$

It can be seen from (6) that the probability of $i$ users being silent is determined completely by $\rho$ and is not influenced by the individual values of $N_{R}$ and $N_{C}$.

In Fig. 3, the reservation process is illustrated. The state variable is the number of users in reservation. Given the number of users in the silence state, the numbers of users in contention and in reservation are dependent. Since there are at most $N$ users in the reservation state at any time instant, given $N_{S}$ silent users, the maximum number of users in reservation, $N^{\prime}$, satisfies

$$
N^{\prime}=\min \left\{N, M-N_{S}\right\}
$$

Let $b(r, s)$ and $d(r)$ denote the birth and death rates of the reservation process, respectively, given $N_{R}=r$ and $N_{S}=s$. Considering the capture effect, they can be evaluated by

$$
\begin{aligned}
d(r)= & \left(\gamma_{f}+P_{R C}\right) \cdot P(\text { current slot is occupied }) \\
= & {\left[\gamma_{f}+\sigma\left(1-\gamma_{f}\right)\right] \frac{r}{N} } \\
b(r, s)= & P\left(\text { one user is captured } \mid N_{R}=r, N_{S}=s\right) \\
& \cdot P(\text { current slot is available }) \\
= & \sum_{k=1}^{c}\left(\begin{array}{l}
c \\
k
\end{array}\right) P_{t}^{k}\left(1-P_{t}\right)^{c-k} P_{c a p t}(k)\left(1-\frac{r}{N}\right)
\end{aligned}
$$

where $P_{t}$ is the permission probability to transmit and $c=$ $M-r-s . P_{c a p t}(k)$ denotes the capture probability given $k$ simultaneous transmissions, which will be evaluated in the next section. Given $N_{S}$, we can solve the Markov chain of the reservation process exactly [4]

$$
P\left(N_{R}=r \mid N_{S}=s\right)=\frac{\prod_{l=0}^{r-1} b(l, s)}{1+\sum_{r=1}^{N^{\prime}}\left[\frac{\prod_{l=0}^{r} b(l, s)}{\prod_{l=1}^{r} d(l)}\right]} .
$$

The unconditional probability that the system has $N_{R}$ users in the reservation state and $N_{S}$ users in the silence state is given by

$P\left(N_{R}=r, N_{S}=s\right)=P\left(N_{R}=r \mid N_{S}=s\right) P\left(N_{S}=s\right)$.

\section{Determination of Dropping Probability}

The unconditional dropping probability will be expressed as [7]

$$
\begin{aligned}
P(\text { dropping })= & \sum_{r=0}^{N} \sum_{c=0}^{M-r} P\left(\text { dropping } \mid N_{R}=r, N_{C}=c\right) \\
& \cdot P\left(N_{R}=r, N_{C}=c\right) \\
= & \sum_{r=0}^{N} \sum_{c=0}^{M-r} P\left(\text { dropping } \mid N_{R}=r, N_{C}=c\right) \\
& \cdot P\left(N_{R}=r, N_{S}=M-r-c\right)
\end{aligned}
$$

In this equation, $P\left(N_{R}=r, N_{S}=M-r-c\right)$ can be evaluated from (10). To find the unconditional dropping probability $P$ (dropping), we need to calculate the conditional dropping probability $P$ (dropping $\mid N_{R}, N_{C}$ ) next.

We illustrate a talkspurt in Fig. 4. The shaded areas denote the packets dropped due to unsuccessful contentions. The dark areas denote the packets with transmission errors. Since transmission errors are uniformly distributed, we can divide the whole talkspurt into several identical cycles except the last 


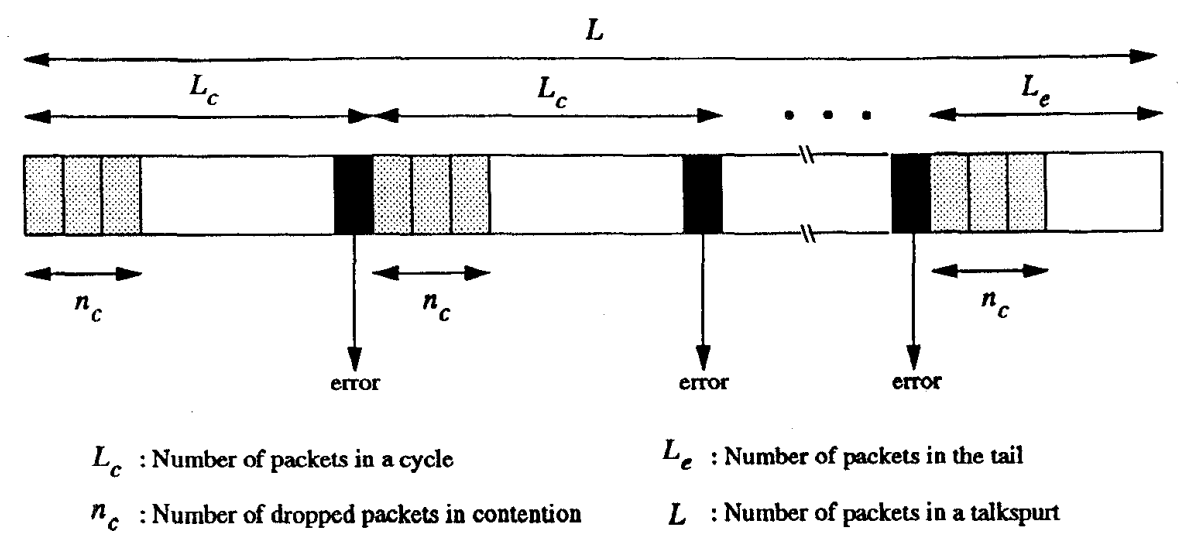

Fig. 4. Illustration of a talkspurt.

several packets, which we call the tail. Each complete cycle ends with a packet that has a transmission error, and the tail ends because of the termination of the talkspurt. Define $n_{d r o p}$ as the number of dropped packets in a talkspurt. Define $n_{c}$ and $n_{e}$ as the number of dropped packets in a cycle due to unsuccessful contentions and transmission errors, respectively. Let $L, L_{c}$, and $L_{e}$ represent the packet length, the cycle length, and the tail length, respectively. Then

$$
P\left(\text { dropping } \mid N_{R}, N_{C}\right) \triangleq \frac{\mathbf{E}\left(n_{d r o p} \mid N_{R}, N_{C}\right)}{\mathbf{E}(L)} .
$$

If we ignore the tail, the dropping probability can be approximated by

$$
\begin{aligned}
P\left(\text { dropping } \mid N_{R}, N_{C}\right) & \approx \frac{\mathbf{E}\left(n_{c} \mid N_{R}, N_{C}\right)}{\mathbf{E}\left(L_{c}\right)}+\frac{\mathbf{E}\left(n_{e} \mid N_{R}, N_{C}\right)}{\mathbf{E}\left(L_{c}\right)} \\
& \triangleq P_{d c}+P_{d e}
\end{aligned}
$$

Here, $P_{d c}$ and $P_{d e}$ denote the dropping probabilities due to unsuccessful contentions and transmission errors, respectively. We evaluate $P_{d c}$ first.

1) Determination of Dropping Probability Due to Unsuccessful Contentions: From [7], we have

$$
\mathbf{E}\left(n_{c} \mid L_{c}, N_{R}, N_{C}\right)=v^{D} \frac{1-v^{N L_{c}}}{1-v^{N}}
$$

where $D$ is the delay limit of voice packets in time slots. $v$ is defined as the probability that a new user with permission to transmit in the current slot does not gain the reservation. Given $N_{R}=r$ and $N_{C}=c$, considering the fading and capture effect, $v$ can be evaluated by

$$
\begin{aligned}
v= & 1-\left(1-\frac{r}{N}\right) P_{t} \sum_{k=0}^{c}\left(\begin{array}{l}
c \\
k
\end{array}\right) \\
& P_{t}^{k}\left(1-P_{t}\right)^{c-k} P_{c a p t}(k+1) .
\end{aligned}
$$

The distribution of $L_{c}$ is needed to evaluate $\mathbf{E}\left(n_{c} \mid N_{R}, N_{C}\right)$. We know that the cycle length $L_{c}$ satisfies the geometric distribution with parameter $\left(1-\gamma_{f}\right)(1-\sigma)$ since a cycle will not terminate unless an error occurs or the talkspurt ends. Then

$$
P\left(L_{c}\right)=\left[1-\left(1-\gamma_{f}\right)(1-\sigma)\right]\left[\left(1-\gamma_{f}\right)(1-\sigma)\right]^{L_{c}-1}
$$

with the average cycle length

$$
\mathbf{E}\left(L_{c}\right)=\frac{1}{1-\left(1-\gamma_{f}\right)(1-\sigma)} .
$$

From (14) and (16)

$$
\begin{aligned}
\mathbf{E}\left(n_{c} \mid N_{R}, N_{C}\right) & =\sum_{L_{c}=1}^{\infty} \mathbf{E}\left(n_{c} \mid L_{c}, N_{R}, N_{C}\right) P\left(L_{c}\right) \\
& =\frac{v^{D}}{1-\left(1-\gamma_{f}\right)(1-\sigma) v^{N}} .
\end{aligned}
$$

The dropping probability due to contention is

$$
P_{d c}=\left[1-\left(1-\gamma_{f}\right)(1-\sigma)\right] \frac{v^{D}}{1-\left(1-\gamma_{f}\right)(1-\sigma) v^{N}} .
$$

Since we are ignoring the tail of the talkspurt, this formula will give pessimistic results. The deviation will be large when the packet header error rate and the number of active users are both small. This is because when $\sigma$ is small, the ratio of $L_{e}$ to $L$ will be large and ignoring this part will cause inaccuracy. This phenomenon can be seen in the results of Section V.

2) Determination of Dropping Probability due to Transmission Errors: Now, we will find $P_{d s}$. We know that if an error occurs, the user will return to the contention state immediately. Hence, the erroneous packet always appears at the end of a cycle. A transmission error is not equivalent to the corresponding packet being dropped. Before it is discarded, the erroneous packet may retry several times until its delay limit $D$ is reached. Here, it is assumed that $D$ is an integral multiple of $N$. Let $K$ be the number of times this erroneous packet tries to retransmit in its lifetime. $K$ satisfies the binomial distribution with

$$
P(K)=\left(\begin{array}{l}
D \\
K
\end{array}\right) P_{t}^{K}\left(1-P_{t}\right)^{D-K} .
$$

The probability that all these $K$ attempts fail is $v^{K}$. Here, we assume for simplicity that $v$ is a constant during the lifetime of a packet. Since the delay limit $D$ is small, it can be assumed that the system state (the values of $N_{R}$ and $N_{C}$ ) does not change too much in this interval, i.e., $v$ is almost a constant. Therefore, if an error indeed occurs, the dropping probability due to transmission errors can be evaluated by

$$
P_{\text {de }}(\text { errors occur, } K \text { attempts })=\sigma v^{K} \text {. }
$$




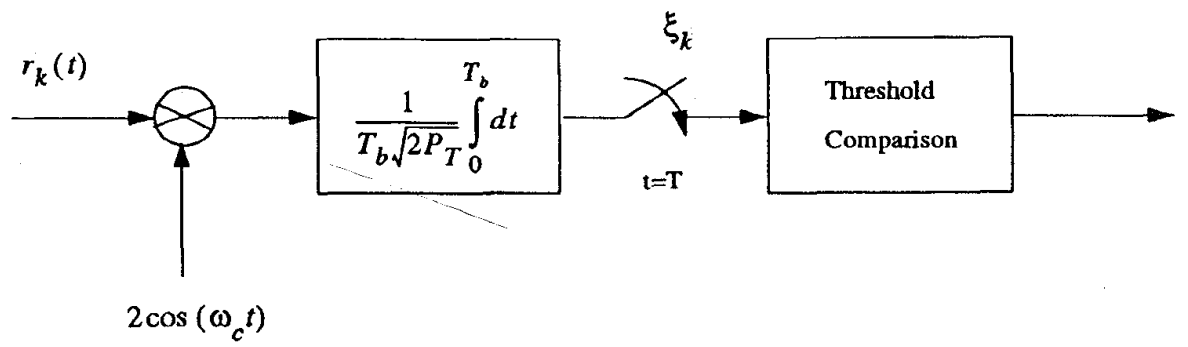

Fig. 5. Correlation receiver for coherent detection of BPSK.

An error may or may not occur after the successful reservation. Denote the number of packets between the successful reservation and the first occurrence of an error as $N_{e} . N_{e}$ follows the geometric distribution with parameter $\sigma$. The number of packets in a talkspurt, $L$, also follows the geometric distribution but with parameter $\gamma_{f}$

$$
\begin{aligned}
P\left(N_{e}\right) & =(1-\sigma)^{N_{e}-1} \sigma, \\
P(L) & =\left(1-\gamma_{f}\right)^{L-1} \gamma_{f} .
\end{aligned}
$$

Only when $N_{e} \leq L$ may an error occur. This probability can be evaluated by

$$
\begin{aligned}
P\left(N_{e} \leq L\right) & =\sum_{L=1}^{\infty} P\left(N_{e} \leq L \mid L\right) P(L) \\
& =\frac{\sigma}{1-(1-\sigma)\left(1-\gamma_{f}\right)} .
\end{aligned}
$$

With the distribution of $K, P_{d e}$ can be calculated from (21) and (23)

$$
P_{d e}=\frac{\sigma^{2}}{1-\left(1-\gamma_{f}\right)(1-\sigma)}\left[1-P_{t}+v P_{t}\right]^{D} .
$$

Therefore, the total conditional dropping probability due to both unsuccessful contentions and transmission errors is given by

$$
\begin{aligned}
& P\left(\text { dropping } \mid N_{R}, N_{C}\right)= \\
& \frac{\sigma^{2}}{1-\left(1-\gamma_{f}\right)(1-\sigma)}\left[1-P_{t}+v P_{t}\right]^{D} \\
& \quad+\left[1-\left(1-\gamma_{f}\right)(1-\sigma)\right] \frac{v^{D}}{1-\left(1-\gamma_{f}\right)(1-\sigma) v^{N}} .
\end{aligned}
$$

Note that if we set

$$
\begin{aligned}
& P_{\text {capt }}(1)=1, \\
& P_{\text {capt }}(k)=0, \quad \forall k>1
\end{aligned}
$$

in (15) and $\sigma=0,(25)$ corresponds to the model without capture in the perfect environment. Equation (25) can be reduced to the formula derived in [7]

with

$$
P\left(\text { dropping } \mid N_{R}, N_{C}\right)=\gamma_{f} \frac{v^{D}}{1-\left(1-\gamma_{f}\right) v^{N}}
$$

$$
v=1-\left(1-\frac{r}{N}\right) P_{t}\left(1-P_{t}\right)^{c}
$$

If we set

$$
\begin{aligned}
& P_{c a p t}(1)=1-\sigma \\
& P_{c a p t}(k)=0, \quad \forall k>1
\end{aligned}
$$

and $\sigma \neq 0$, we obtain the model without capture but with transmission errors. In this case, it is found in Section $\mathrm{V}$ that the analytical results given by (25) show close agreement with the computer simulations given in [3].

\section{CAPTURE MODEL}

To study the capture effect on the PRMA system performance, we need to develop the capture model first. In this work, the capture model focusing on the data link layer is employed. It is assumed that the receiver randomly locks onto a favorable packet and acquires perfect bit synchronization to it. If the detection occurs without error, this packet is assumed to be captured [5].

For simplicity, the normalized circular cell model is used instead of a hexagonal one. The base station is at the center of the cell. Users with omnidirectional antenna are uniformly distributed. We assume that the frequency reuse distance is large enough so that the co-channel interference can be ignored. Also, it is assumed that the near-far effect is compensated (by power control, ${ }^{1}$ etc.). The signals transmitted from different users to the base station experience slow flat Rician fading with lognormal shadowing independently. BPSK modulation is employed, and a coherent detector is used at the receiver (Fig. 5). Since higher error rate is tolerable in voice communications, we assume that as long as the receiver decodes the header of a packet without error, this packet is received correctly. Let the header be $H$ bits long.

Let $I$ be the number of simultaneous transmissions in a cell. With the slow fading assumption, the effect of fading on all the bits throughout the whole packet is the same. Therefore, the received signal for each arbitrary bit has an identical form as follows [8]:

$$
\begin{aligned}
r(t)= & r_{0}(t)+\sum_{i=1}^{I-1} r_{i}(t)+n(t) \\
= & \sqrt{2 P_{T}} \alpha_{0} l_{0} \cos \left(\omega_{c} t\right) \\
& +\sum_{i=1}^{I-1} \sqrt{2 P_{T}} \alpha_{i} l_{i} \cos \left(\omega_{\mathrm{c}} t+\theta_{i}\right)+n(t)
\end{aligned}
$$

where $P_{T}$ is the transmitted power and $n(t)$ is the Additive White Gaussian Noise with two-sided power spectrum density $\mathcal{N}_{0} / 2$. Subscript " 0 " stands for the tagged packet and " $i$ " for

\footnotetext{
'Note that power control is used to compensate for the near-far effect, so as to make the performance location independent. The application of power control will weaken the capture effect.
} 


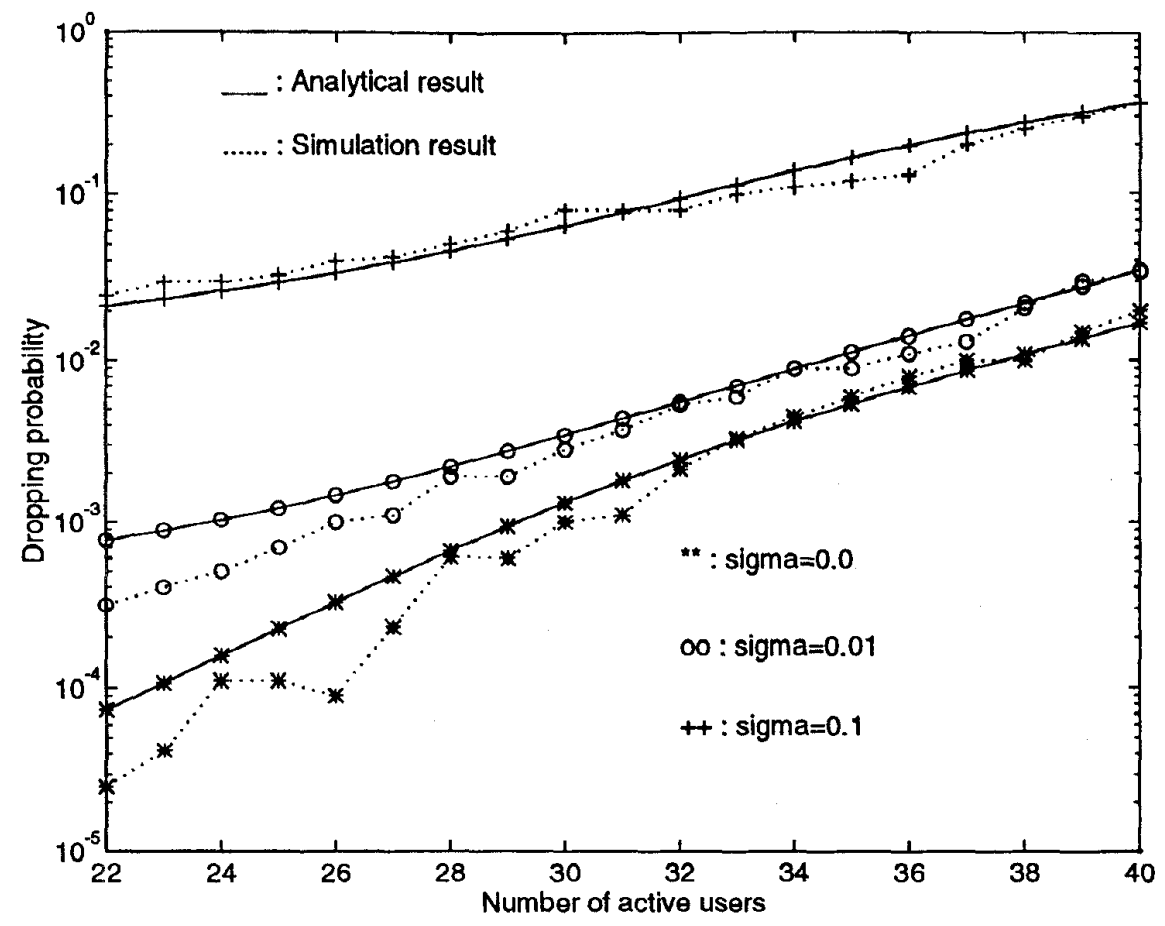

Fig. 6. Dropping probability in fading channels, without capture.

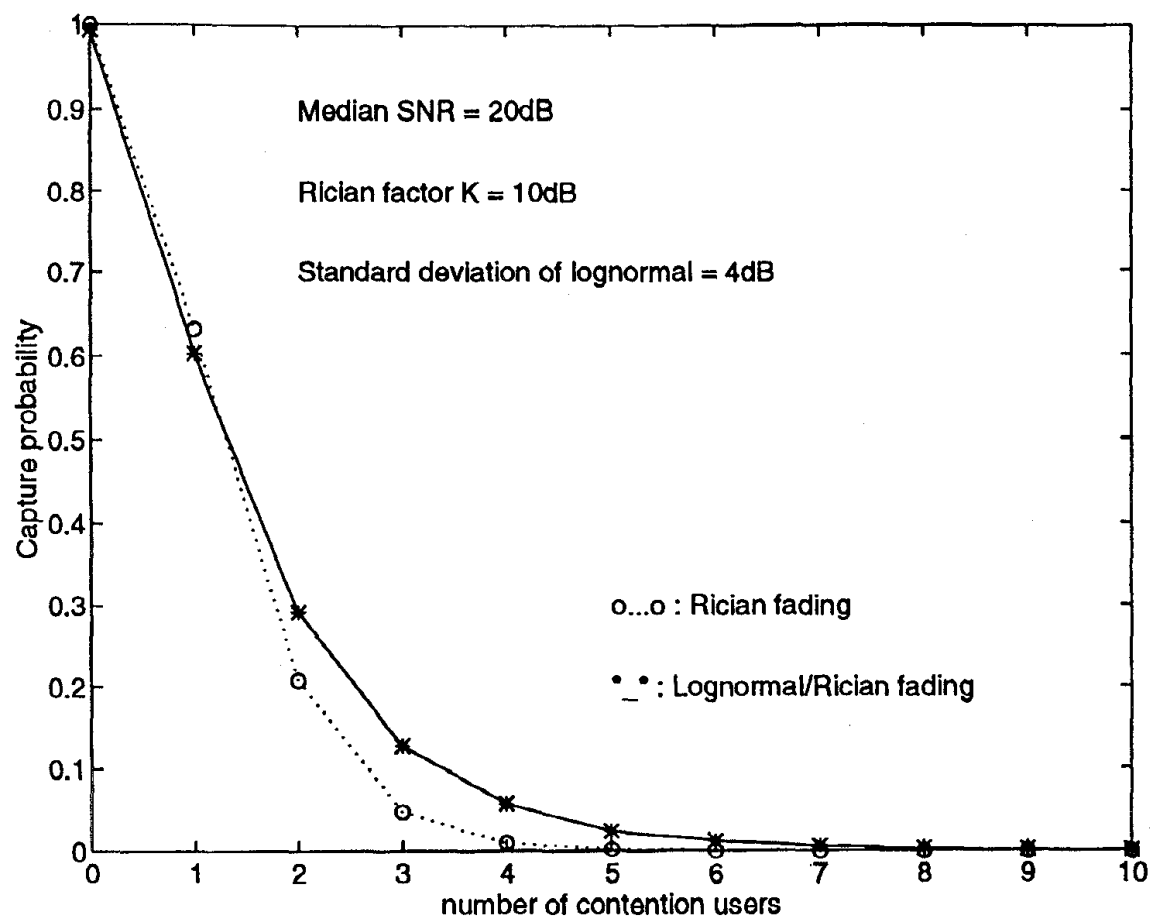

Fig. 7. Capture probability versus the number of contention users.

the $i$ th contention packet. $\alpha_{i}$ is the bit pattern of packet $i$, be expressed as taking on values \pm 1 with equal probability. $\theta_{i}$ is the phase offset of the $i$ th contention packet relative to the desired packet. $l_{i}$ accounts for the fading and shadowing, which can

$$
l_{i}=\sqrt{\mathcal{L}_{i}} f_{i}
$$

where $\mathcal{L}_{i}$ and $f_{i}$ are random variables following the lognormal 
TABLE I

SYSTEM PARAMETERS

\begin{tabular}{|l|l|}
\hline \hline Parameter & Numerical Example \\
\hline channel rate, $R_{c}$ Kbps & 720 \\
\hline source rate, $R_{s}$ Kbps & 32 \\
\hline PRMA frame duration, $T \mathrm{~ms}$ & 16 \\
\hline number of slots per frame, $N$ & 20 \\
\hline slot duration, $\tau$ ms & 0.8 \\
\hline number of overhead bits per packet, $H$ bits & 64 \\
\hline speech delay constraint, $D$ slots & 40 \\
\hline average duration of talkspurts, $t_{1} \mathrm{~s}$ & 1.0 \\
\hline average duration of silence gaps, $t_{2} \mathrm{~s}$ & 1.35 \\
\hline median SNR, dB & 20 \\
\hline \hline PRMA permission probability, $P_{t}$ & variable \\
\hline number of active users in a cell, $M$ & variable \\
\hline
\end{tabular}

and Rician distribution, respectively. $\mathbf{E}\left(f_{i}^{2}\right)=1$. For analytical simplicity, it is assumed that time delays are zero and the receiver can perfectly track the phase of the desired user. With coherent detection, the decision variable will be

$$
\begin{aligned}
\xi & =\frac{1}{\sqrt{2 P_{T}} T_{b}} \int_{0}^{T_{b}} r(t) \cdot 2 \cos \left(\omega_{c} t\right) d t \\
& =\alpha_{0} l_{0}+\sum_{i=1}^{I-1} \alpha_{i} l_{i} \cos \left(\theta_{i}\right)+n_{0}
\end{aligned}
$$

where $T_{b}$ is the bit duration and $n_{0}$ is a zero mean Gaussian random variable with variance $\sigma_{n}^{2}=\mathcal{N}_{0} / 2 E_{b} . E_{b}$ is the bit energy. Defining

$$
L_{I}=\sum_{i=1}^{I-1} \alpha_{i} l_{i} \cos \left(\theta_{i}\right)
$$

then

$$
\xi=\alpha_{0} l_{0}+L_{I}+n_{0} .
$$

Let us define random vectors $\underline{l}, \underline{f}, \underline{\mathcal{L}}, \underline{\theta}$, and $\underline{\alpha}$ as $I$-component vectors of the form $\underline{l}=\left(l_{0}, \cdots, l_{I-1}\right)^{T}$ and similarly for the rest. Using BPSK, the bit error rate (BER) of the desired user given $I-1$ contention packets is

$$
\begin{aligned}
P_{b}(I \mid \underline{l}, \underline{\theta}, \underline{\alpha}) & =P\left(\xi<0 \mid \alpha_{0}=1\right) \\
& =Q\left(\frac{l_{0}+L_{I}}{\sigma_{n}}\right)
\end{aligned}
$$

where $Q(x)=1 / \sqrt{2 \pi} \int_{x}^{\infty} e^{-y^{2} / 2} d y$. If $\underline{l}$ and $\underline{\theta}$ are given, the average conditional BER can be obtained by averaging over all the possible data patterns $\underline{\alpha}$

$$
\bar{P}_{b}(I \mid \underline{l}, \underline{\theta})=\mathbf{E}_{\underline{\alpha}}\left\{P_{b}(I \mid \underline{l}, \underline{\theta}, \underline{\alpha})\right\}
$$

With the slow fading assumption, $\bar{P}_{b}(I \mid \underline{l}, \underline{\theta})$ will be identical for all the $H$ bits in a packet header. Therefore, the conditional capture probability is

$$
P_{\text {capt }}(I \mid \underline{l}, \underline{\theta})=\left[1-\bar{P}_{b}(I \mid \underline{l}, \underline{\theta})\right]^{H}
$$

To evaluate the unconditional capture probability by multifold integration will be computationally expensive, although we know the probability distributions of $\underline{l}$ and $\underline{\theta}$. Therefore, Monte Carlo summation is employed to calculate the capture probability approximately. Note that if we set $I=1$, the packet header error rate $\sigma$ can be evaluated using the same formula

$$
\begin{aligned}
P_{\text {capt }}(I) & =\mathbf{E}_{\underline{l}} \mathbf{E}_{\underline{\theta}}\left\{P_{\text {capt }}(I \mid \underline{l}, \underline{\theta})\right\} \\
& \approx \lim _{Q \rightarrow \infty} \frac{1}{Q} \sum_{j=1}^{Q} P_{c a p t}\left(I \mid \underline{l}_{j}, \underline{\theta}_{j}\right) .
\end{aligned}
$$

\section{NUMERICAL RESUlTS}

To facilitate the comparison of our analytical results with the simulation [3], we use the same system parameters as in [3] (Table I).

The dropping probability formula in a fading environment (25) is verified by comparing the results with those from simulations [3], setting

$$
\begin{aligned}
& P_{\text {capt }}(1)=1-\sigma, \\
& P_{\text {capt }}(k)=0, \quad \forall k>1
\end{aligned}
$$

and $\sigma=0,0.01,0.1$. The result is shown in Fig. 6, where the simulation results given by [3] are duplicated. It is found that our analytical results fit the simulation results very well. However, since we ignore the tail of the talkspurt when we derive the formula, the results are pessimistic especially when the tail is not small compared to the length of the talkspurt.

The capture probabilities in Rician and lognormal/Rician channels are evaluated using Monte Carlo summation. The results are shown in Fig. 7 . It is found that if $M$ is small, the capture probability in Rician channel is larger than that in the shadowed Rician channel. However, the capture probability in Rician channel will decrease more quickly. 


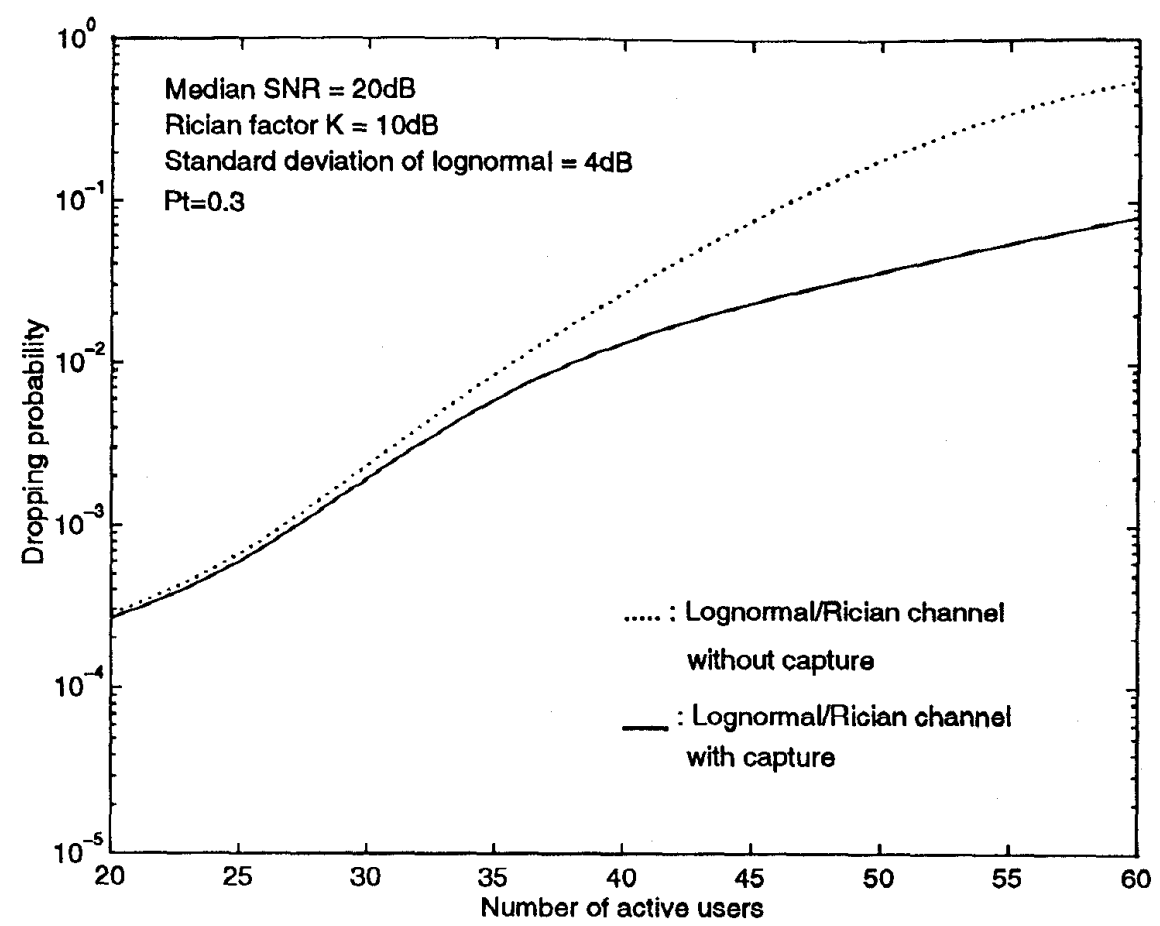

Fig. 8. Dropping probability in the lognormal/Rician fading channel, $P_{t}=0.3$.

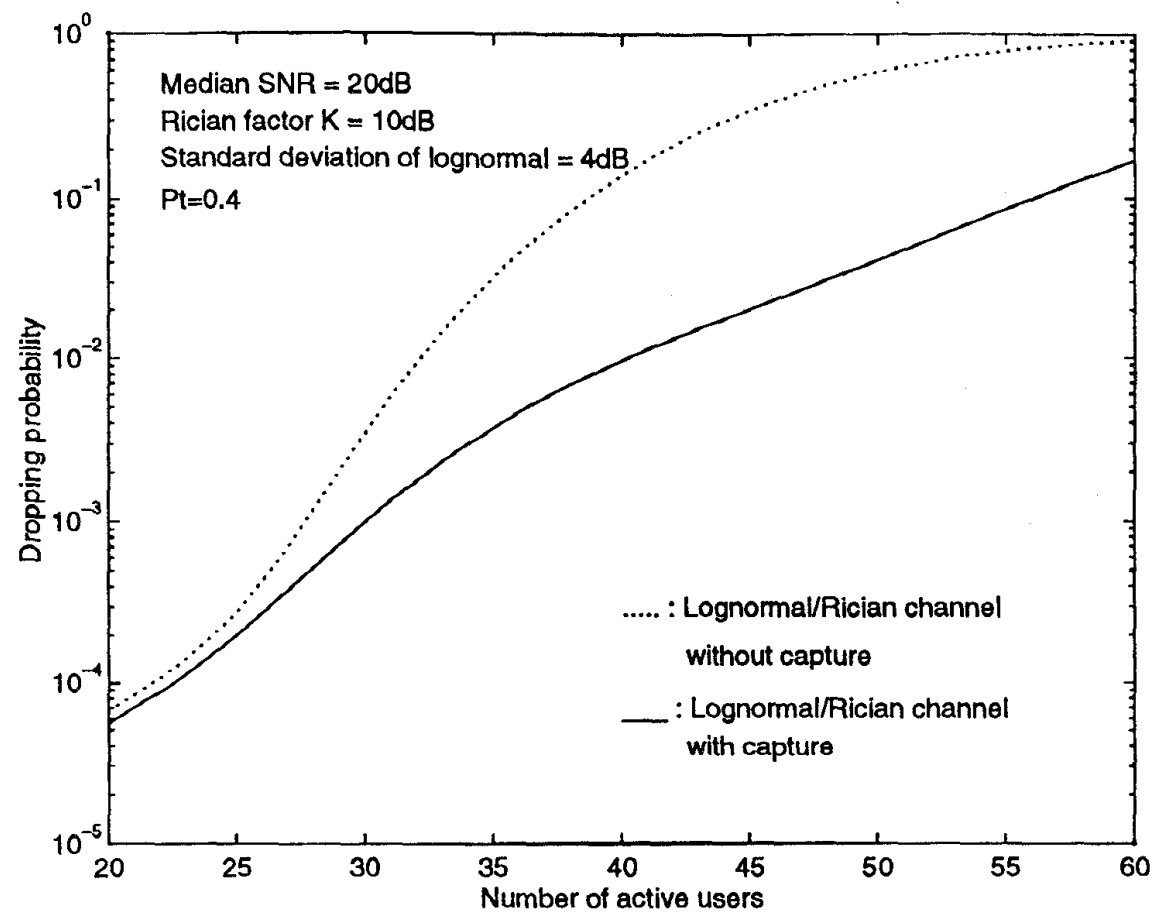

Fig. 9. Dropping probability in the lognormal/Rician fading channel, $P_{t}=0.4$.

We illustrate the dropping probabilities in the lognormal/Rician channel in Figs. 8 and 9 with $P_{t}=0.3$ and $P_{t}=0.4$, respectively. It is found that with capture, the user capacity is improved and the service quality degrades gracefully as $M$ increases, compared to the traditional system without capture.

Fig. 10 illustrates how the dropping probability will be influenced by the permission probability. If $P_{t}$ is too small, 


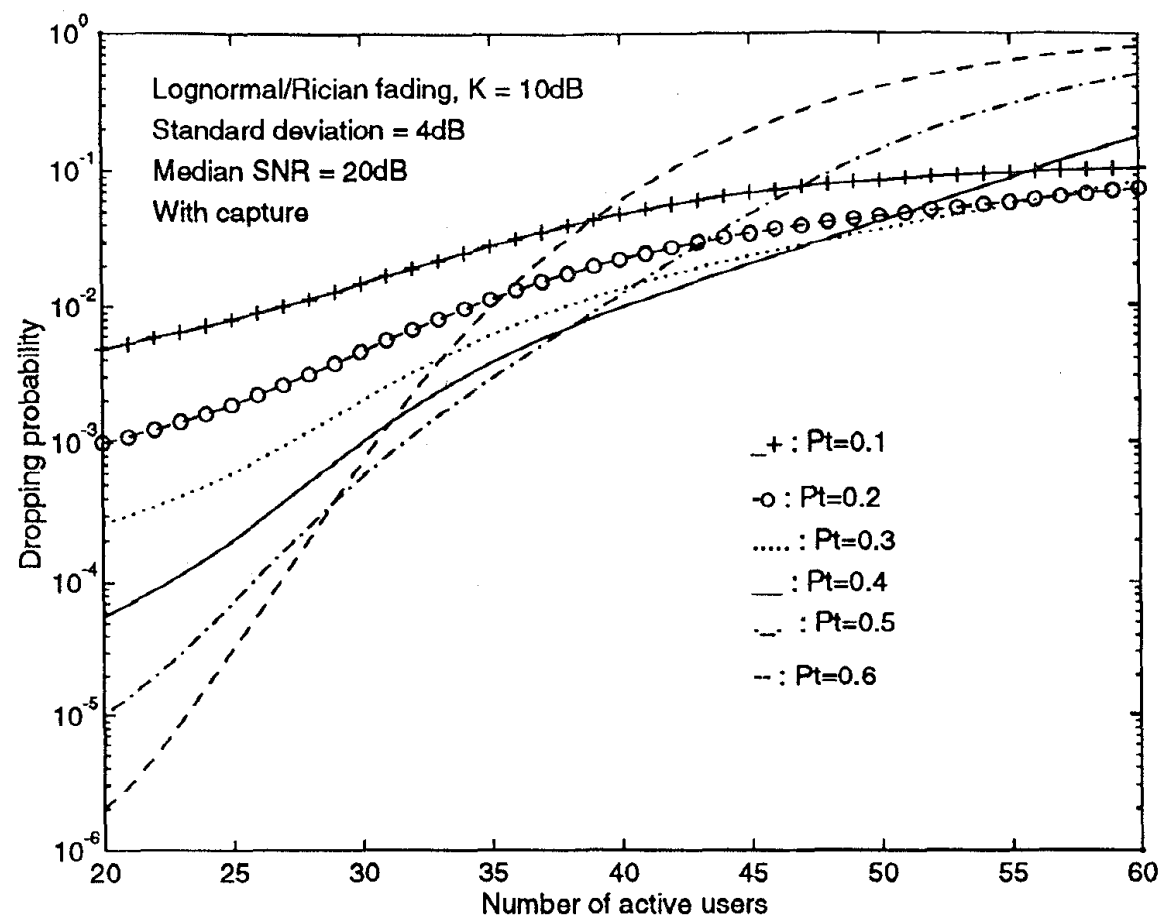

Fig. 10. Effect of permission probability on the dropping probability in the lognormal/Rician fading channel.

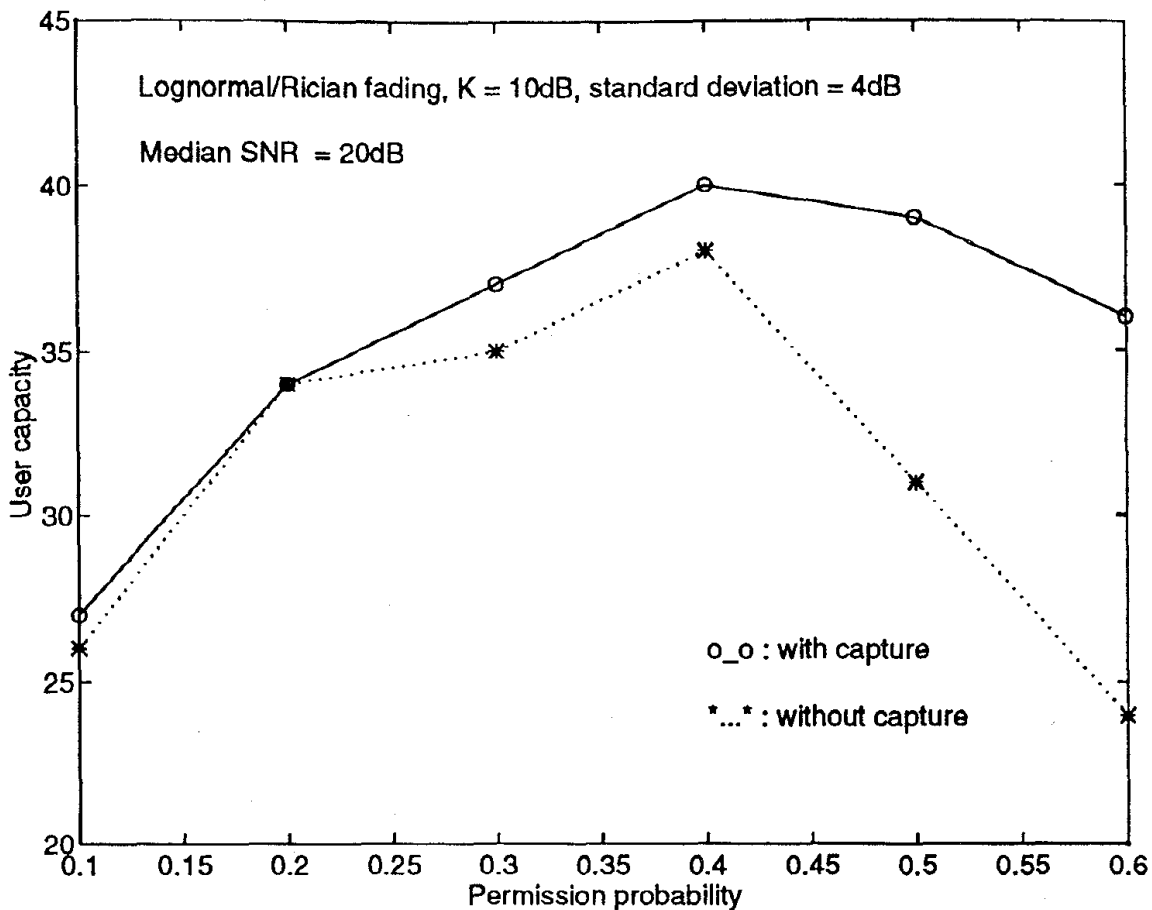

Fig. 11. Capacity versus permission probability in the lognormal/Rician fading channel.

more packets will be dropped due to fewer chances to transmit. If $P_{t}$ is too large, the dropping probability will increase very rapidly because more collisions occur. The relationship between the user capacity and the permission probability is shown in Fig. 11. Here, we set the dropping probability criterion to be $1 \%$. It is found that $P_{t}=0.3$ is the best choice considering both the user capacity and the performance degradation. 


\section{CONCLUSION}

In this paper, we develop a Markovian model to analyze PRMA systems in a microcellular environment. A new formula is derived to evaluate the dropping probability in fading channels. The accuracy of this formula is verified by simulation. The dropping probability is studied by this formula accounting for both the capture and the fading effects. The optimal permission probability is found. The numerical results show that the desirable soft capacity feature is exhibited in the PRMA system with capture.

\section{ACKNOWLEDGMENT}

The authors would like to thank Dr. C.-m. Sun for his valuable comments.

\section{REFERENCES}

[1] D. J. Goodman, R. A. Valenzeula, K. T. Gayliard, and B. Ramamurthi, "Packet reservation multiple access for local wireless communications," IEEE Trans. Commun., pp. 885-889, Aug. 1989.

2] D. J. Goodman and S. X. Wei, "Efficiency of packet reservation multiple access," IEEE Trans. Veh. Technol., vol. 40, no. 1, pp. 170-180, Feb. 1991.

[3] L. M. A. Jalloul, S. Nanda, and D. J. Goodman, "Packet reservation multiple access over slow and fast fading channels," in IEEE VTC' 90 1990 , pp. $354-359$.

[4] L. Kleinrock, Queueing Systems-Volume I: Theory. Wiley, 1975.

[5] J.-P. M. G. Linnartz, R. Hekmat, and R.-J. Venema, "Near-far effects in land mobile random access networks with narrow-band Rayleigh fading channels," IEEE Trans. Veh. Technol, vol. 4l, no. 1, pp. 77-90, Feb. 1992.

[6] N. M. Mitrou, TH. D. Orinos, and E. N. Protonotarios, "A reservation multiple access protocol for microcellular mobile-communication systems," IEEE Trans. Veh. Technol., vol. 39, no. 4, pp. 340-351, Nov. 1990.

[7] S. Nanda, D. J. Goodman, and U. Timor, "Performance of PRMA: A packet voice protocol for cellular systems." IEEE Trans. Veh. Technol. vol, 40, no. 3, pp. 584-598, Aug. 1991.

[8] C. Sun, "Packet radio communications in a microcellular environment," Ph.D. dissertation, Univ, of Southern California, 1994.

[9] C. Wang, J. Wang, and B. Sukhbaatar, "Performance evaluation of PRMA system with channel fading," in IEEE ICC'93, 1993, pp. 1952-1954.

[10] K. Zhang and K. Pahlavan, "Relation between transmission and through put of slotted ALOHA local packet radio networks," IEEE Trans. Commun., vol. 40, no. 3, pp. 577-583, Mar. 1992.

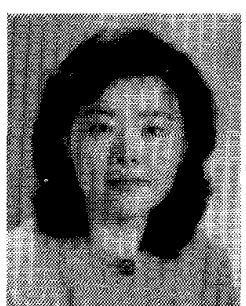

communications.
Xiaoxin Qiu (M'96) received the B.E. and M.E. degrees from Tsinghua University, People's Republic of China, in 1990 and 1991, respectively, and the Ph.D. degree from the Department of Electrical Engineering of the University of Southern California Los Angeles, in 1996.

She recently joined AT\&T Research Laboratory, working in the Broadband Wireless Systems Re search Department. Her research interests are in the areas of wireless communications networks, personal communication systems, and multimedia

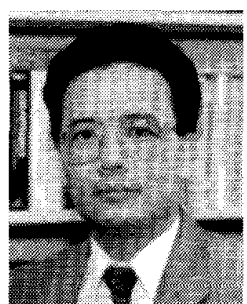

Victor O. K. Li (S'80-M'81-SM'86-F'92) was born in Hong Kong in 1954. He received the S.B. S.M., and Sc.D. degrees in electrical engineering and computer science from the Massachusetts Institute of Technology, Cambridge, MA, in 1977, 1979, and 1981, respectively.

Since February 1981 he has been with the University of Southern California (USC), Los Angeles, where he is Professor of Electrical Engineering and Director of the USC Communication Sciences Institute. His research interests include high-speed communication networks, personal communication networks, multimedia systems, distributed databases, queueing theory, graph theory, and applied probability. He has lectured and consulted extensively around the world.

Dr. $\mathrm{Li}$ chaired the Computer Communications Technical Committee of the IEEE Communications Society from 1987 to 1989 and the Los Angeles Chapter of the IEEE Information Theory Group from 1983 to 1985 . He is the Steering Committee Chair of the International Conference on Computer Communications and Networks $\left(\mathrm{IC}^{3} \mathrm{~N}\right.$ ), General Chair of the first Annual IC $^{3}$ N, June 1992, Technical Program Chair of the Institution of Electrical Engineers (IEE) Personal Communication Services Symposium, June 1995. and Chair of the Fourth IEEE Workshop on Computer Communications, October 1989. A member of ACM, he has served as an Editor of IEEE Network and of Baltzer Telecommunication Systems, Guest Editor of IEEE JourNaL ON SElected AREAS in COMmunications and of Elsevier Computer Networks and ISDN Systems, and is now serving as an Editor of ACM Wireless Networks. 